\title{
Pervasive refusal syndrome or anorexia nervosa: a case report with a successful behavioural treatment
}

\author{
A. Perrone ${ }^{1}$ - S. F. Aruta ${ }^{1}$ - G. Crucitti ${ }^{2} \cdot$ P. Gualandi ${ }^{2} \cdot$ E. Malaspina ${ }^{2} \cdot$ M. Marino ${ }^{3} \cdot$ E. Franzoni $^{1}$. \\ Antonia Parmeggiani ${ }^{1}[\mathbb{C}$
}

Received: 3 August 2020 / Accepted: 12 August 2020 / Published online: 20 August 2020

(c) Springer Nature Switzerland AG 2020

\begin{abstract}
Purpose Pervasive refusal syndrome (PRS) is a rare psychiatric disease that affects children. It was first described by Lask in 1991 (Arch Dis Child 66:866-869, 1991). Recently, Otasowie and Collaborators reported a systematic review about PRS. Despite this, PRS has not yet been classified in DSM-5 and ICD-11 and the lack of evidence-based treatment makes this syndrome a real challenge for clinicians. The aim of this paper is to present our experience through the description of a case report and its treatment.

Methods and results The case reported is a girl aged 11 years that fits the clinical picture described in the literature of PRS. In previous reports, behavioural treatment was not used or appreciated; our case adds new knowledge regarding the PRS diagnosis and the successful behavioural treatment during hospitalization, which we describe in all its phases.

Conclusion PRS is a rare, life-threatening syndrome; it would be extremely important to have an official and evidence-based treatment guide.

Level of evidence Level V, case report.
\end{abstract}

Keywords Pervasive refusal syndrome $\cdot$ Eating disorders $\cdot$ Feeding disorders $\cdot$ Children $\cdot$ Behavioural treatment $\cdot$ Case report

\section{Introduction}

The pervasive refusal syndrome (PRS) was first described by Lask in 1991 [1]. A recent, systematic review of the literature by Otasowie and Collaborators in 2020 [2] shows the high attention paid to this issue. The disorder is rare but potentially life threatening. The DSM-5 and ICD-11 do not recognize PRS among classified mental disorders. Jaspers et al. suggested these diagnostic criteria in 2009 [3]:

Antonia Parmeggiani

antonia.parmeggiani@unibo.it

1 Regional Centre for Feeding Eating Disorders, Child Neurology and Psychiatry Unit, Department of Medical and Surgical Sciences, S. Orsola Malpighi Hospital, University of Bologna, Bologna, Italy

2 Regional Centre for Feeding and Eating Disorders, Child Neurology and Psychiatry Unit, S. Orsola Malpighi Hospital, Bologna, Italy

3 Clinical Nutrition and Metabolism Unit, S. Orsola Malpighi Hospital, Bologna, Italy
1. Partial or complete refusal in three or more of the following domains: eating, mobilization, speech, and attention to personal care.

2. Active and angry resistance to acts of help and encouragement.

3. Social withdrawal and school refusal.

4. No organic condition accounts for the severity of the degree of symptoms.

5. No other psychiatric disorder could better account for the symptoms.

6. The endangered state of the patient requires hospitalization.

A multifactorial theory has been described to explain the aetiology of PRS, including predisposing factors (premorbid personality, usually perfectionist and high-achieving), precipitating factors (traumatic events perceived as out of control) and perpetuating factors (parent's and doctor's reactions). Enmeshed mother-child relationship $[4,5]$ and parental psychiatric problems (not otherwise specified) are both frequent [6]. 
Excluding organic causes or possible acute threats to survival remains a priority. The prescribed examinations are almost always normal. Many different diagnoses are usually attempted before the right one is identified (especially, depression and eating disorders).

No specific therapy is available for this syndrome, nor evidence-based treatment has been presented in the literature. Treatment must be multidisciplinary and must include the following mainstays: patience, empathy, and time; nursing care by skilled staff; use of ward milieu; physiotherapy; individual therapy; parental counselling/family therapy [5, 6]. It seems that drugs play a limited role. In the first period of the illness, enteral or parenteral feeding is almost always necessary. In previous reports, behavioural treatment was not used or appreciated [7], classical cognitive-behavioural programs seem to be useless; in particular, children appear not to respond to punishments and disincentives [4]. Despite this, some articles report the success of a cognitive-behavioural setting and the possibility of influencing the patient's behaviour if $s / h e$ is informed that $s / h e$ would consequently get what $\mathrm{s} / \mathrm{he}$ most wants (for example, the patient is told that $\mathrm{s} / \mathrm{he}$ will be permitted something if s/he starts feeding him/ herself) $[8,9]$. Indeed, the aim of this article is to present an Italian case of PRS and its behavioural treatment.

\section{Case report}

G., an 11-year-old girl, was hospitalized in our Regional Centre for feeding and eating disorders with an eating disorder and selective mutism. G.'s parents gave signed informed consent to the processing of personal data at the time of the clinical evaluation.

G. was previously hospitalized in a Paediatric Unit, where organic causes or possible acute threats to survival had been excluded; so, the primary concern of the staff regarded the diagnostic challenge. Physical examination at admission showed a weight of $24.9 \mathrm{~kg}$, a BMI of $13.4 \mathrm{~kg} / \mathrm{m}^{2}$; dehydration and pale skin were evident; she had a nose-gastric feeding tube. The communication, mobilization, eye contact and facial mimic were practically absent; she used to speak only to her parents. Her parents' high-conflict separation led to her shared custody shortly after birth. Family history was negative for psychiatric pathology. The girl's physical and intellectual development had been normal. G. was a good student at school; she had no close friends, she had never gone to birthday's parties or played sports; she was not autonomous or self-driven (e.g., she did not dry her hair or go to school alone). G.'s eating pattern had always been selective: when she was 8 years old, she had an indigestion after which she began a qualitative restriction of food (only yogurt, meat, vegetables and very few carbohydrates). On her eleventh birthday, she started a quantitative restriction and, during the last months before the hospitalization, she almost completely quit eating. Moreover, she expressed an increasing refusal to go to school. G.'s first period under our care was characterized by a pervasive refusal affecting all aspects of her life: she spent her days in a foetal position, covering her eyes with her hands, constantly groaning and rejecting any offer to help. Diagnostic tests (blood count, electrolytes, liver and kidney function, blood sugar, protein, thyroid hormones, vitamin D, vitamin B12, iron, electrocardiogram) were all normal. It was not possible to give G. self-administered questionnaires due to her total lack of collaboration. Once, when cousins, uncle and aunt came to visit her, G. moved without any help from her room to another, spoke normally and played cards; she stopped when staff members arrived, as happens in patients with selective mutism. G.'s mother was a really difficult person to cooperate with: her relationship with her daughter perfectly fits the literature's description of a mother-child enmeshed relationship and of the "loss of the internal parent", or "theory of learnt hopelessness" [7]. She always indulged her daughter by seconding her requests and opposed all the proposals of the caring staff. She played down her daughter's progress while, at the same time, claiming that G. was able to do something when, in fact, she was not. However, even the mother was poorly independent: she always needed to be accompanied by her sister or a family member to the hospital. G.'s father, instead, was more positive and collaborative and played an important role in G.'s rehabilitation process. G. even made some progress when her father assisted her in the ward and at the prospect of receiving permission to go to his house over the weekend, whereas she tended to lose these feeding acquisitions when her mother returned to assist her.

\section{Treatment}

The aims of the treatment were to improve G.'s self-government through progressive accomplishments in three different domains: eating behaviours, daily autonomies and social skills. To assess the three main domains, we used a three-branch system: quality of the performance (QP), independence/assistance (I/A) and self-determination (SD). For each branch, we created different subdomains consisting of specific goals (e.g., "eating", "walking"). Finally, we assigned a score to each goal from 0 (total lack of collaboration) to 5 (autonomous and normal gesture or expression) (see Table 1). We observed G. for $1 \mathrm{~h}$ twice a week in the three different contexts. First, we performed a skill baseline evaluation using a specific checklist that we created for every competence area. Then we introduced cognitive-behavioural strategies like prompting, fading, modelling and the task analysis (see Fig. 1).

G. and her parents attended individual, marital and family therapy and took part in group sessions. Moreover, once G. 
Table 1 Results: eating behaviours, daily autonomies, and social skills

\begin{tabular}{|c|c|c|c|c|c|c|}
\hline & \multicolumn{2}{|l|}{ QP } & \multicolumn{2}{|l|}{$\mathrm{I} / \mathrm{A}$} & \multicolumn{2}{|l|}{ SD } \\
\hline & 1st week & Last 2 weeks & 1st week & Last 2 weeks & 1st week & Last 2 weeks \\
\hline \multicolumn{7}{|l|}{ Eating behaviours } \\
\hline Agree to eat & 0 & 5 & 0 & 5 & 0 & 5 \\
\hline Agree to drink & 0 & 5 & 0 & 5 & 0 & 5 \\
\hline Chewing properly & 0 & 5 & 0 & 5 & 0 & 5 \\
\hline Using cutlery correctly & 0 & 3 & 0 & 3 & 0 & 4 \\
\hline Using glass correctly & 0 & 4 & 0 & 4 & 0 & 4 \\
\hline Using the napkin correctly & 0 & 4 & 0 & 3 & 0 & 3 \\
\hline \multicolumn{7}{|l|}{ Daily autonomies } \\
\hline Taking two steps & 0 & 5 & 0 & 5 & 0 & 5 \\
\hline Walking & 0 & 5 & 0 & 5 & 0 & 5 \\
\hline Getting out of bed & 0 & 5 & 0 & 4 & 0 & 4 \\
\hline Sitting on the chair & 0 & 5 & 0 & 4 & 0 & 4 \\
\hline Opening/closing the door & 0 & 3 & 0 & 3 & 0 & 3 \\
\hline Switching on/off the light & 0 & 4 & 0 & 4 & 0 & 4 \\
\hline \multicolumn{7}{|l|}{ Social skills } \\
\hline Keeping attention on the interlocutor & 0 & 5 & 0 & 5 & 0 & 5 \\
\hline Maintaining eye contact & 0 & 5 & 0 & 5 & 0 & 5 \\
\hline Replying with a nod & 0 & 5 & 0 & 5 & 0 & 5 \\
\hline Maintaining physical contact & 0 & 4 & 0 & 5 & 0 & 5 \\
\hline Manifesting emotions with facial expressions & 0 & 4 & 0 & 4 & 0 & 4 \\
\hline Communicating by gestures & 0 & 5 & 0 & 5 & 0 & 4 \\
\hline Negotiating solutions & 0 & 3 & 0 & 3 & 0 & 4 \\
\hline
\end{tabular}

$Q P$ quality of the performance, $I / A$ independence/assistance, $S D$ self-determination

Fig. 1 Treatment and results: timelines of eating behaviours and daily autonomies

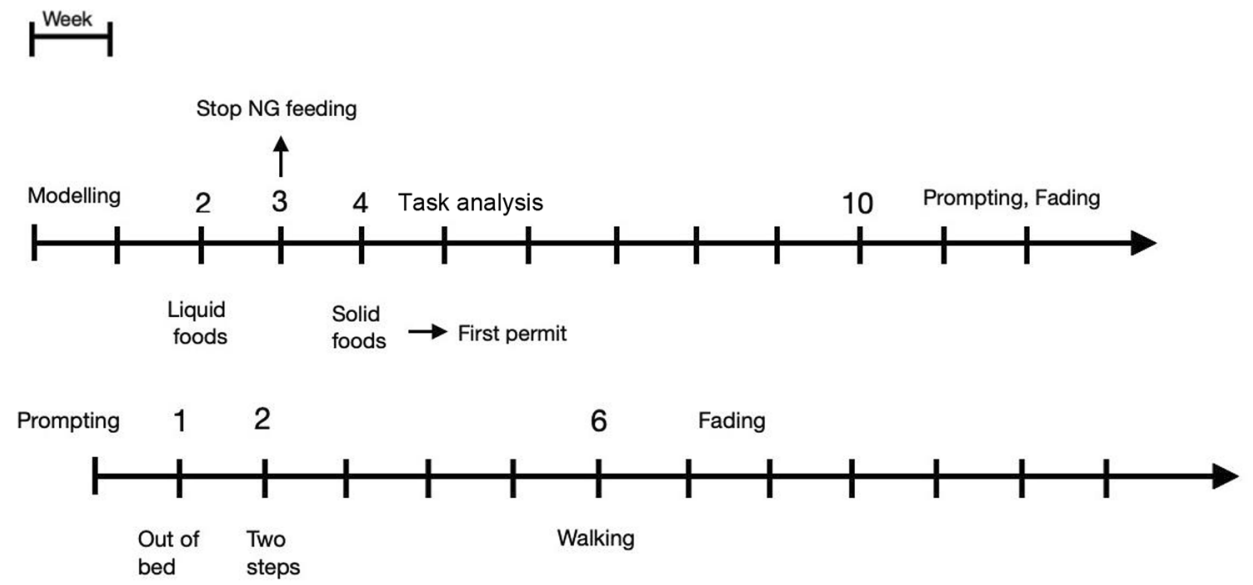

no longer needed the NGT, we made a deal with the family: G. would spend the weekend at home if she reached the goal of the week and kept the previously achieved ones.

Staff members including a child neuropsychiatrist, a paediatrician, a dietician, and psychologists always gave G. appropriate verbal instructions. As a physical prompt, for example, we touched her arm when we wanted her to use the fork, and continued to touch her arm until she reached her mouth and pulled the fork back. Meanwhile,
G.'s mother held her other hand during each bite. Later on, we introduced fading, that is, a gradual decrease of provided aid (less touch surface, less pressure). Concerning social skills, we mostly used gradual exposure in vivo: staff members always maintained rich communication through stories, music, schooling and games; they continued to do so despite G.'s resistance. The treatment lasted for 23 weeks. We repeated the initial observations during the last 2 weeks (see Table 1). 
At the time of hospital discharge, G. was not completely autonomous while feeding. Nevertheless, eating behaviours received the highest scores. The independence of the girl received the lowest scores, and this is probably due to the behaviour of her mother. Regarding daily autonomies, self-determination was not brilliant because G. needed our encouragement many times. So, at the time of discharge, G. did not have a full recovery. However, the short duration of hospitalization ( 23 weeks) compared to that reported in the literature (87 weeks) must be considered [3, 10]. At the time of the hospital discharge, the staff decided, after reporting the case to the Juvenile Justice Court, to entrust the girl to the territorial service of child and adolescent neuropsychiatry, to proceed with a daily psycho-educational program at home with an educator. At present, G. is 13 years old, she is regularly menstruating and eats restrictive categories of food. During the COVID-19 emergency, she completed her studies at home. She does not want to go out and stays with her mother all day long.

\section{Discussion}

G. never manifested the typical thoughts connected with eating disorders, in particular with anorexia nervosa. On the contrary, she presented a selective mutism compatible with social anxiety, and all these aspects are described as possible clinical features of PRS. The main differential diagnosis at the beginning of her hospitalization, which is worth discussing, was of avoidant/restrictive food intake disorder (ARFID), particularly of food avoidance emotional disorder (FAED): affected children show an apparent lack of interest in food which leads to significant weight loss; there is no evidence of a disturbance in the way in which one's body weight or shape is experienced; malnutrition is associated with marked interference with psychosocial functioning. Nevertheless, in PRS, the refusal is, by definition, pervasive in all areas and is accompanied by an important rejection of any offer of help. ARFID seems to be a wider and less homogeneous disorder, and could underlie organic and functional disorders, while PRS appears to be a distinct nosographic entity with a purely psychic etiopathogenesis.

The treatment of patients affected by PRS comes with a very high human and economic cost $[3,10]$. Focusing on our case, since pressuring patients usually aggravates their condition [3] and therapeutic enthusiasm is almost always counterproductive $[3,6]$, we established few achievable goals for G. Regular meetings were not enough to avoid discussions and misunderstandings with G.'s mother; she always tried to re-negotiate and manipulate objectives [10]. Indeed, it is absolutely necessary to acknowledge that the major, if not the only, problem during G's treatment was her mother's behaviour, and that such condition persists during the clinical evolution.

The strength of our work lies in the diagnosis and description of the treatment in each of its phases and in the evaluation-as objective and reproducible as possible-of the results achieved in each area. However, the major limitations consist of the lack of ability to generalize and a limited support of scientific literature.

\section{Conclusions}

PRS is a life-threatening syndrome that should have an official and evidence-based treatment guide.

The aim of the authors was to report a new Italian case, paying particular attention to its multidisciplinary treatment based on teamwork and collaboration with other services. Behavioural treatment can be successful in PRS; in previous reports it was not used or appreciated.

\section{What is already known on this subject?}

PRS is a rare psychiatric disease that affects children, which has not yet been included in the official diagnostic manuals and does not have an evidence-based treatment.

\section{What do we now know as a result of this study that we did not know before?}

Through our case report, we described a successful behavioural treatment in all its aspects and procedures. This could be particularly useful and interesting to those physicians and caregivers who face the challenges posed by the treatment of this complex psychiatric pathology.

Funding Not applicable.

\section{Compliance with ethical standards}

Conflict of interest The authors declare that there is no conflict of interest.

Ethical approval For case reports, approval from the ethics committee is not required.

Informed consent G.'s parents gave signed informed consent to the processing of personal data at the time of the clinical evaluation.

Availability of data and material Not applicable.

Code availability Not applicable. 


\section{References}

1. Lask B, Britten C, Kroll L, Magagna J, Tranter M (1991) Children with pervasive refusal. Arch Dis Child 66:866-869. https://doi. org/10.1136/adc.66.7.866

2. Otasowie J, Paraiso A, Bates G (2020) Pervasive refusal syndrome: systematic review of case reports. Eur Child Adolesc Psychiatry. https://doi.org/10.1007/s00787-020-01536-1

3. Jaspers T, Hanssen GMJ, van der Valk JA, Hanekom JH, van Well GTJ, Schieveld JNM (2009) Pervasive refusal syndrome as part of the refusal-withdrawal-regression spectrum: critical review of the literature illustrated by a case report. Eur Child Adolesc Psychiatry 18(11):645-651. https://doi.org/10.1007/s00787-009-0027-6

4. Thompson SL, Nunn KP (1997) The pervasive refusal syndrome: the RAHC experience. Clin Child Psychol Psychiatry 2(1):145165. https://doi.org/10.1177/1359104597021010

5. Guirguis S, Reid C, Rao S, Grahame V, Kaplan C (2011) Followup study of four cases of pervasive refusal syndrome. Eur Child Adolesc Psychiatry 20(5):271-274. https://doi.org/10.1007/s0078 7-011-0169-1
6. Lask B (2004) Pervasive refusal syndrome. Adv Psychiatr Treat 10:153-159. https://doi.org/10.1192/apt.10.2.153

7. Nunn KP, Thompson SL (1996) The pervasive refusal syndrome: learned helplessness and hopelessness. Clin Child Psychol Psychiatry 1(1):121-132. https://doi.org/10.1177/135910459601101 1

8. Wright B, Beverley D (2012) Pervasive refusal syndrome. Clin Child Psychol Psychiatry 17(2):221-228. https://doi. org/10.1177/1359104511403680

9. Bond EC, Oliphant RYK (2018) Pervasive refusal syndrome in autistic spectrum disorder. Case Rep Psychiatry 2018:5049818

10. Nunn KP, Thompson SL, Moore SG, English M, Burke EA, Byrne N (1998) Managing pervasive refusal syndrome: strategies of hope. Clin Child Psychol Psychiatry 3(2):229-249. https://doi. org/10.1177/1359104598032007

Publisher's Note Springer Nature remains neutral with regard to jurisdictional claims in published maps and institutional affiliations. 